\title{
Une mobilisation continuée
}

A continuous mobilisation

\section{Alain Froidevaux}

\section{OpenEdition}

\section{Journals}

Édition électronique

URL : https://journals.openedition.org/ccs/326

DOI : $10.4000 /$ ccs.326

ISSN : 2558-782X

\section{Éditeur :}

Presses universitaires de Rennes, Association des lecteurs de Claude Simon

\section{Édition imprimée}

Date de publication : 8 juillet 2016

Pagination : 111-133

ISBN : 978-2-7535-4876-3

ISSN : 1774-9425

\section{Référence électronique}

Alain Froidevaux, « Une mobilisation continuée », Cahiers Claude Simon [En ligne], 11 | 2016, mis en ligne le 15 septembre 2017, consulté le 21 septembre 2021. URL : http://journals.openedition.org/ccs/ 326 ; DOI : https://doi.org/10.4000/ccs.326 


\title{
UNE MOBILISATION CONTINUÉE
}

\author{
Alain FROIDEVAUX \\ Collège de Genève
}

Quod vitae sectabor iter? etc...

Ausone

Mais qu'est-ce que le voyage procure au lecteur? Quand donc est-il pris à ce point par la lecture et sent-il l'existence de son héros mêlée à la sienne propre avec autant de certitude? Son corps n'est-il pas la navette qui, à la cadence des roues, passe inlassablement à travers la chaîne, le livre du destin de son héros?

Walter Benjamin

En date du 2 mars 1983, dans un essai de plan d'un roman encore à l'état de "vague - très vague - projet ", entre autres " thèmes " retenus par Claude Simon (un "réserviste", un "brigadier", un "prisonnier», un " voyageur ", " la recherche du corps de [son] père »?), la "mobilisation " se trouve épinglée comme l'un des fils de ce chantier alors provisoirement intitulé "Complément » ou "Complément d'information ${ }^{1}$ ". Cette " catégorie du monde militaire comprenant les processus critiques par lesquels des potentiels de combats sont portés à la disponibilité ${ }^{2}$ " avait en son temps déjà

1. Voir "Essai de plan pour L'Acacia» dans Claude Simon, Euvres, Gallimard, "La Pléiade ", 2013, t. 2, p. 1364-1367. L'Acacia sera cité dans cette édition sans autre précision que la pagination. Pour les autres romans (précisés par leurs initiales), les références seront prises dans les deux volumes de l'édition Pléiade (2006 pour le t. 1).

2. Dans la perspective d'une réflexion critique de la modernité, Peter Sloterdijk, donne un tour conceptuel à la notion commune de mobilisation en reprenant la question de l'autoposition du sujet (La Mobilisation infinie, vers une critique cinétique de la cinétique politique, trad. H. Hildebrand, Christian Bourgois, 2000, p. 42). Pour ma part, c'est dans une perspective poétique que je me propose d'examiner l'extension de ce terme. 
été relatée dans $L a$ Corde raide par l'écrivain, mais de manière factuelle, avec les affiches officielles posées le 26 août 1939 pour convoquer les réservistes. Quels compléments d'informations, la reprise d'un tel motif dans L'Acacia viendrait-elle apporter au dossier déjà bien documenté d'une œuvre placée sous le signe d'une " belligérance généralisée ${ }^{3}$ " ?

"Le premier choc de la mobilisation » intervient dans le troisième chapitre (27 août 1914) avec la mise sur pied de guerre d'un régiment appelé à être quasiment décimé dès le début des hostilités. L’onde de choc revient tout au long du sixième chapitre de cette grande polyphonie romanesque «à base de vécu " lors du déplacement d'un jeune réserviste dilettante vers son centre d'incorporation, le 27 août 1939. Ainsi que dans le " théâtre " du septième chapitre (1982-1914) où le départ du père pour la guerre prend l'allure d'une ekphrasis tragique motivée par le témoignage de deux vieilles parentes de l'écrivain. Dans une pièce de l'antique demeure familiale hantée par le portrait d'un ancêtre légendaire (le tableau sanglant de La Route des Flandres?), tel Hector faisant ses adieux à Andromaque et à son fils Astyanax, un capitaine à barbe carrée et à moustaches en crocs, prend congé de sa famille réunie, "disant simplement d'une voix enjouée, calme, comme s'il allait prendre le train ou l'omnibus: "Eh bien voilà! C'est l'heure. Allons" "(p. 1141). Joignant le geste à la parole, déposant son tout jeune fils dans les bras d'une servante noire, le père mobilisé se met en marche pour monter au front où il tombera le 27 août 1914. La forme impérative et la force illocutoire de la dernière parole d'un revenant résonnent comme une convocation ${ }^{4}$. Et, dans le huitième chapitre, c'est encore par une action mémorable que s'affichera en toutes lettres la mobilisation générale sur une manchette de journal (p. 1155).

"Écrire n'est pas raconter ses souvenirs, ses amours et ses deuils, ses rêves et ses fantasmes ", pense Gilles Deleuze, qui poursuit: " la littérature suit la voie inverse, et ne se pose qu'en découvrant sous les apparentes personnes la puissance d'un impersonnel qui n'est nullement une généralité, mais une singularité au plus haut point ${ }^{5} \ldots$ » Nul doute que l'écrivain ne puisse faire sienne

3. Selon la formule de Michel Thouillot, Les guerres de Claude Simon, PUR, «Interférences », 1998, p. 19 sq.

4. C'est en tout cas ce terme de " convocation " qui vient à l'esprit de Claude Simon lorsqu'il cherche à décrire le jeu de la langue dans son travail d'écrivain, voir "Claude Simon à la question " dans Jean Ricardou (dir.), Lire Claude Simon, Colloque de Cerisy, UGE, 1975, p. 428 et p. 418.

5. Gilles Deleuze, "La littérature et la vie ", Critique et clinique, Minuit, p. 12-13. On retrouve une idée analogue dans les notes de lecture de Merleau-Ponty: "on ne lit plus Je ou il/ Il nait des personnes intermédiaires, une $1^{\mathrm{e}}-2^{\mathrm{e}}$ personne " (Maurice Merleau-Ponty, "Cinq notes sur Claude Simon », Esprit, $\mathrm{n}^{\circ} 66$, juin 1982 , p. 65). 
cette thèse du philosophe. Cette singulière impersonnalité n'était-elle pas déjà revendiquée par le bavard de La Corde raide ${ }^{6}$ ? Avec Alastair Duncan, nous voyons bien comment " le moi se dissout - pour se construire en parlant ${ }^{7}$ ", tout au long de cette forme d'autoportrait d'un dormeur éveillé. Entre les temps du passé employés par la remémoration dans les premières pages et le récit au présent des dernières, ce n'est donc plus tout à fait le même esprit qui se trouve affecté par la pensée tourbillonnante des choses lui traversant sans arrêt la tête (bruits, couleurs, odeurs, véhicules bondés de voyageurs qui lisent le journal du soir...). Se souvenir et s'en tenir à la simple relation de ce que l'on a pu voir n'est alors plus vraiment d'actualité dans la dernière partie de ce texte. En réalisant que toute la confuse matière d'une vie ("paroles, actes, pensées, et les fumées de tous les trains [qu'il a] pris, les costumes [qu’il a] portés, l'odeur des gares ") glisse " entre les lignes de la mémoire ${ }^{8}$ ", tel un poisson de la main qui cherche à le saisir, c'est non seulement l'authenticité et l'objectivité du témoignage mais, plus essentiellement encore, c'est la possibilité même d'une remémoration qui se trouvent mise en cause. "À cause de tout ça, je ne suis pas moi » en vient-il à conclure en modifiant une formule rimbaldienne: "Je est un autre". Pas vrai: "Je est d'autres?." "

Vincent Descombes, dans son essai sur Proust, philosophie du roman, note ceci: "Une idée quelconque devient une "idée de roman" lorsque l'écrivain a trouvé le moyen de l'“analyser" ${ }^{10}$. "Il précise: " nous cherchons ici la philosophie du roman, non dans telle ou telle idée, mais dans ce travail lui-même. "Et s'il y a lieu de comparer l'activité de certains romanciers à celle du philosophe, c'est lorsque cette activité se trouve motivée par « l'intention d'éclaircir ce qui était resté obscur ${ }^{11}$ (les pensées fuyantes, les sentiments confus, les situations paralysantes) ». Mais il convient, ajoute-t-il, de distinguer clairement le philosophe et le romancier puisque « la philosophie cherche à éclaircir des pensées par la voie d'un examen des propositions dans lesquelles ces pensées sont communiquées ", alors que c'est dans le travail du

6. Ce qualificatif, le narrateur de La Corde raide le prend lui-même à son compte: « Un bavard c'est un type qui parle de tout ce qui peut s'exprimer au moyen de mots, et un tableau c'est un type qui parle de ce qui ne peut rentrer en l'homme que par les yeux ", La Corde raide, Sagittaire, 1947, p. 72.

7. Alastair B. Duncan, "Claude Simon: le projet autobiographique", Revue des sciences humaines, $\mathrm{n}^{\circ} 220,1990$, p. 53.

8. Claude Simon, La Corde raide, Éd. du Sagittaire, 1947, p. 173.

9. Ibid., resp. p. 170 et 174 .

10. Vincent Descombes, Proust, philosophie du roman, Minuit, 1987, p. 90.

11. Ibid., p. 71. 
style que le romancier s'efforcera de réaliser cette intention. C'est ainsi que la pensée romanesque conçue comme recherche d'éclaircissement revient "à chercher dans cela même qui a demandé du travail au romancier ${ }^{12}$ ". Dans la poétique simonienne, le travail est la règle. Quant aux vertus analytiques $\mathrm{du}$ labeur stylistique, l'écrivain lui-même pourrait y souscrire lorsqu'il propose, par exemple, cette formule (qu'il attribue à Cocteau) : «le style, c'est la façon la plus simple qu'un auteur a trouvée pour dire des choses compliquées ${ }^{13}$ ».

Le devenir autre d'un moi n'est-il au fond pas plus complexe à effectuer que ne le laisse entendre la formule rimbaldienne modifiée de La Corde raide? Subsiste une certaine obscurité dans la reformulation de cette proposition. N'illustre-t-elle pas l'un de ces "mots d'auteur" comme on dit au théâtre? C'est-à-dire, selon Simon, une réflexion étrangère à la littérature, une "scorie » que l'artiste doit scrupuleusement faire en sorte d'éliminer? En reprenant l'idée d'un moi réserviste appelé à devenir brigadier, prisonnier et, peut-être même écrivain, l'écriture de L'Acacia procéderait à cet effort de clarification.

\section{LES TRAINS DE LA MOBILISATION}

Avant d'être générale, la mobilisation commence par prendre forme au début du sixième chapitre (le 27 août 1939), avec l'entrée en gare d'une locomotive " aérienne et monumentale [...], comme portée sur un nuage » (p. 1101). Le chapitre se termine lorsqu'un personnage de réserviste pourra prendre place dans un omnibus. Dans l'intervalle, d'autres moyens de transport se seront articulés à la motrice initiale: voitures de première et de troisième classe, wagons à compartiments, wagons à bestiaux. D'un train l'autre, c'est tout un réseau ferroviaire qui devient dans le texte une gigantesque constellation de tonalité célinienne "où la nuit n'aurait jamais de fin» (p. 1133). Animés par des forces monstrueuses, ces «trains qui tous ensemble, au même moment, grondaient sur des ponts, s'engouffraient dans des tunnels, franchissaient des fleuves, sifflaient lugubrement, haletaient à travers les plaines d'un continent couturé de cicatrices » (p. 1127), réalisent "une espèce de mutation" (p. 1108) au cours de laquelle en moins de vingt-quatre heures les voyageurs passent non seulement " du soleil au froid, mais d'un univers normal [...] à un monde endeuillé, sévère, catégorique "

12. Ibid., p. 46.

13. Francine Mallet (réal.), Écrivains $d u X X^{*}$ siècle: Claude Simon, France Culture, mai 1971 (1 ${ }^{\mathrm{re}}$ diff.). En réalité, cette formule se trouve dans la réponse d'André Maurois au discours de réception à l'Académie française de Cocteau. 
(p. $\left.1134^{14}\right)$. Propédeutique à l'expérience du front et à la violence des combats, le mouvement locomoteur reconfigure les voyageurs en sujets mobilisés. Au début du huitième chapitre, certains d'entre eux se retrouvent devant la grille d'une usine à gaz réaffectée pour l'occasion en centre mobilisateur : " deux cents hommes environ, munis de petites valises ou de musettes, jeunes pour la plupart mais qui ne ressemblaient en rien à ceux qui étaient montés dans le train la veille ou pendant la nuit» (p. 1146). Mais plus précisément à quel événement pourrait nous préparer cette apparition initiale?

Pilotée par un mécanicien et par un chauffeur aux lunettes relevées qui "regardaient de leurs yeux bordés de rouge » la foule pétrifiée sur le quai, l'oxymorique locomotive et ses wagons « en bois, peints d'une couleur marron écaillée ", tracent un "illusoire et ultime espace de vide " entre la foule et le convoi immobilisés, instaurent "comme un fossé, un étroit canyon ou plutôt une invisible muraille, un invisible rempart au-delà duquel, une fois franchi, serait scellé quelque chose d'irrémédiable, définitif et terrible " (p. $1101^{15}$ ). La puissance de ce monstre tellurique imprime à la description sa dynamique. Aussi illusoire soit-il, le sceau n'en demeure pas moins une empreinte que pourra emprunter la démarche (la méthode) d'une écriture travaillant au descellement d'un monument ${ }^{16}$. Prêt à reprendre la route après avoir englouti son lot d'appelés, le convoi s'ébroue " comme s'il cherchait à secouer à la façon d'un animal couvert de parasites les grappes humaines attachées à ses flancs " (p. 1103). Cet animal destructeur couvert de vermine est bien une machine à produire " de l'énergie militaire " (selon une formule de Valéry ${ }^{17}$ ) et son formidable potentiel démiurgique demande à être considéré dans la double perspective d'une narratologie et d'une grammatologie.

Changement de focale avec la mise en route du convoi : c'est en compagnie des voyageurs arrachés à leurs proches restés en arrière que nous éprouvons la dérive et le naufrage d'un monde familier: "puis même plus des visages: des taches - puis même plus des taches: des points - puis même plus des points: rien qu'un indistinct et sombre agrégat sur le quai de la gare miniature "

14. Voir l'interprétation faite par Marie-Christine Mourier du mot "catégorique " comme hapax simonien en relation avec l'expérience du "réel " dans "Du mot catégorique dans Les Géorgiques (1981) de Claude Simon ", Cahiers Claude Simon, n 9, PUR, 2014, p. 85-96.

15. Sauf mention contraire ici et dans la suite, c'est nous qui soulignons.

16. L'antique demeure familiale, que le réserviste laisse derrière lui, pourra être décrite comme un " obscur mausolée" (p. 1232).

17. Cité par Wolfram Nitsch dans «Techniques de la métaphore et métaphores techniques chez Claude Simon ", dans I. Albers et W. Nitsch (dir.), Transports, les métaphores de Claude Simon, Francfort, Peter Lang, 2006, p. 245. 
(p. 1106). À chaque halte où l'opération se répète, "l'on pouvait de nouveau entendre le halètement régulier de locomotive" (p. 1105). Désormais, c'est l'impersonnalité du pronom de la troisième " personne » qui semble prévaloir dans ce transport. Tel un "silencieux cyclone », celui-ci laisse « derrière lui un monde intact mais vide, inhabité, à l'exception ici et là de quelques poules errant au hasard, comme aveugles, affairées et manchotes entre les brancards des carrioles et les charrues échouées" (ibid.). Cette exception appelée à devenir la règle se vérifie sur les vitres des wagons où s'animent l'image d'un paysage crépusculaire et les «doubles immatériels et immobiles des occupants assis ou couchés sur les banquettes, les journaux à gros titres déchirés et étalés sous eux " (p. 1108). Dans le train de la mobilisation, la divagation hasardeuse de ces volatiles aveugles et manchots (prise au pied de la lettre) correspond à l'actualité des titres des manchettes des quotidiens ${ }^{18}$ (voire même à la couture de chevrons sur la manche d'une vareuse militaire).

Emportés par le mouvement du train, les hommes subissent silencieusement les cahots (l'actualisation du chaos), cependant que l'un ou l'autre ramasse machinalement "quelque exemplaire froissé d'un journal du jour » (p. 1106). Mais à quoi bon s'intéresser à la lecture de quotidiens : ils répètent tous le même titre "en lettres énormes ", "une simple dilatation typographique de mots que les journaux avaient déjà imprimés, ou plutôt que postulait l'ensemble des mots imprimés par les journaux (mais en caractères plus petits) depuis déjà plusieurs semaines - en fait, depuis plusieurs mois - en fait, depuis plusieurs années" (ibid.). L'épanorthose et le " comme si » caractéristiques du style simonien permettent d'identifier la dilatation typographique comme une crise de la syntaxe assurant « un ordre pour ainsi dire de bienséante immunité " aux mots de la tribu ; comme un signe symptomatique d'une époque où le temps semble être sorti de ses gonds. Contaminés par l'hybris en cours, les mots des manchettes se développent démesurément et leur excroissance pathogène laisse alors penser (entre parenthèses, comme en passant) qu'ils « avaient perdu toute leur raison d'être, la syntaxe expulsée elle aussi, les manchettes (les manchettes qui dans les jours à venir allaient être suivies de plusieurs autres de taille chaque fois croissante, jusqu'à ce qu'enfin les lettres remplissent la moitié de la page) réduites à l'assemblage

18. Dans Les Géorgiques, sur la moquette du bureau où $\mathrm{O}$. essaye de rédiger son témoignage de brigadiste en subodorant la vanité de toute tentative de témoignage, se trouvent des journaux "à demi déployés, froissés, s’affalant comme des espèces de volatiles aux ailes grisâtres, cassées, inertes » ( $G$, II, p. 870). Dans la description d'une peinture prête à s'animer de Leçon de choses (p. 559), ce sont déjà les plumes de ces volatiles qui font le coup de pinceau, la griffe du peintre. 
de deux ou trois substantifs isolés et démesurément agrandis " (p. 11061107). De manière parfaitement rigoureuse, la description d'un phénomène typographique permet tout à la fois de diagnostiquer une déconstruction syntaxique comme une crise générale de l'intelligence et de recycler ces « lettres de deuil » dans la composition du récit. De surcroît, le geste stylistique permet d'ébaucher un personnage. Dans le prolongement de cinq parenthèses emboîtées comme des poupées russes, apparaît un homme sans qualités particulières, un voyageur comme les autres. Emporté par le mouvement général, cet homme ne semble plus trouver grand sens à la lecture des journaux; ou même vouloir jeter un coup d'œil à leurs titres. À ce moment du récit, la conduite de cet individu au journal déployé sur ses genoux n'a pourtant rien d'insignifiant. Installé dans son rôle, le voici donc " les yeux fixes, contemplant sans les voir les caractères imprimés et les photographies elles aussi démesurément agrandies, puis laissant tout tomber» (p. 1107). Aussi retiré dans ses pensées que puisse être le destinataire de signes démesurément agrandis - « comme à l'intention de myopes ou d'idiots " (ibid.) - celui-ci n'en restera pas quitte pour autant. Déchirés, étalés, recyclés (comme papier pour emballer des sandwichs, par exemple), les journaux qu'on laisse tomber, les titres qu'on ne prend même plus la peine de lire ne cessent cependant d'intervenir dans le texte comme des titres de transport ${ }^{19}$. Et à ce titre, ils entraînent le récit à prendre une certaine allure spéculative.

\section{UNE MOBILISATION SINGULIÈRE}

«Et maintenant il allait mourir» (p. 1108). À peine vient-il de prendre pied dans le récit que voici le personnage désœuvré et promis à disparaître. Comment recevoir une telle prédiction? À qui revient-il de pouvoir en énoncer la fatalité? Cette formule lapidaire, où se répercutent voix narrative et parole de personnages, est un cas de monologue narrativisé. "Le monologue narrativisé, remarque Dorrit Cohn, est le médium privilégié de ces instants de suspension temporelle [suspended in an instant present], où un personnage se révèle [for revealing a fictional mind] entre le passé qui resurgit du souvenir et l'anticipation du futur ${ }^{20}$." Cette formule initiale instaure

19. Ce motif revient à cinq reprises, au moins, dans le sixième chapitre (p. 1106, 1107, 1108, 1127 et 1134) et par trois fois dans la huitième partie du roman (p. 1155, 1157 et 1166).

20. Dorrit Cohn, La Transparence intérieure, Modes de la représentation de la vie psychique dans le roman, trad. A. Bony, Le Seuil, coll. « Poétique », 1981, p. 150. 
l'instant présent et ouvre un espace de maintenance dans lequel se configure un esprit (mind) en mouvement.

À mi-parcours, lors d'un changement de train à Lyon, en compagnie d'un autre jeune appelé, c'est dans une voiture de première classe qu'avec ce voyageur plongé dans le noir, nous prendrons peu à peu la mesure de sa conduite ${ }^{21}$. Précédé par le choc de valises cognant contre la paroi, un nouvel arrivant fait irruption dans le compartiment, en principe réservé aux officiers. Dans le contexte de cette nouvelle étape, on trouve une première occurrence du discours rapporté avec l'intrusion du gradé, lequel d'une voix furibonde intime aux jeunes gens de justifier leur présence dans le wagon: "Qui êtes-vous montrez-moi vos papiers où allez-vous?, l'autre garçon répondant toujours sans se lever On va tous au même endroit: au casse pipe [...] Pouvez pas nous laisser dormir non?, la voix sèche, rageuse, indignée disant Dor... Do... Ah par exemple, par ex» (p. 1111). Comme la syntaxe des titres, la voix autoritaire bredouille. Abdiquant, l'officier cède le terrain et le garçon impertinent s'endort presque aussitôt. Quant au témoin de l'altercation, resté dans une distante neutralité (" on entendit») lui, par contre, garde les yeux bien ouverts tout en manifestant une sensibilité vive, une faculté attentionnelle particulière à ce qui (lui) arrive. Il s'imprègne de l'odeur ambiante charbonneuse qui est la senteur même "de tout arrachement et de tout désastre " (p. 1109). Il ressent précisément le « pesant assemblage (roues, essieux, boggies) » du wagon et cette perception esthétique lui permet de se figurer « le vaste et sourd grondement qui monterait du sol lui-même, comme si d'un bout à l'autre de l'Europe la terre obscure était en train de trembler sous les innombrables convois emportés dans la nuit " (p. 1112).

L'une des pièces motrices de l'assemblage fonctionne comme un instrument d'optique, lunette astronomique ou verre correcteur propre à corriger une myopie contemplative, en aidant le voyageur à reconnaître dans le noir la forme (une Gestalt?) de son corps «tout entier gisant à plat sur la banquette "; et, plus exactement encore, à contempler "dans une perspective télescopique [...] les vingt-six années qui maintenant allaient selon toute probabilité trouver une fin»(p. 1109). Deux images saisissantes se réfractent grâce à cette lentille clarificatrice: la tombe paternelle introuvable dans un paysage d'apocalypse et le cercueil de la mère. Ces projections convergent dans l'esprit du sujet, allongé comme dans un cercueil sur la ban-

21. À première vue, une "idiotie narratologique ", si avec Jean Kaempfer on considère "la stricte restriction du point de vue ", comme l'un des trois traits caractéristiques des récits de guerre modernes (Poétique du récit de guerre, Corti, coll. « Les Essais », 1998). 
quette d'un compartiment (une boîte, un coffre scellé). En nous mettant à sa place (mais au juste laquelle, dans ce roman qui s'ingénie à multiplier les lieux et les époques?), nous pourrions considérer qu'un orphelin doté de tels souvenirs ne peut qu'envisager l'issue fatale à son propre déplacement militaire en ce 27 août, date anniversaire de la mort de son père. Cette interprétation psychologique est naturellement envisageable mais (si l'on y tient) ne risque-t-elle pas de nous faire passer un peu vite sur le sens de ce qui est exactement en train de se jouer dans l'esprit d'une personne ou, plus exactement, d'un masque, d'une persona à l'identité problématique? La question du sujet mobilisé - dans et par ce texte - ne relèverait-elle au fond pas d'une solution, d'une chimie plus complexe?

Ainsi, quelle valeur donner à ce "maintenant " de l'énoncé prédictif initial - un adverbe appelé à proliférer (plus d'une quinzaine d'occurrences) dans le texte? Indication narrative de régie ou marque déictique du discours intérieur du personnage? D'une manière ou d'une autre, cette catégorie linguistique occupe l'esprit du jeune réserviste aussi bien que l'acte narratif qui, au "présent de l'écriture ", expose et réfléchit de manière dynamique le mouvement de la pensée d'un sujet mobilisé. Plus qu'à une simple remémoration, le discours « intérieur " donne lieu à une forme de memento mori dans lequel une anaphorique prolifération de "pensa-t-il ", de monologues narrativisés, de citations d'expressions d'humeur mènent au constat que, de cette collection de souvenirs, « ne subsistait plus à présent que l'insignifiant résidu [...] de vingt-six années de paresse et de nonchalante inertie - au mieux, de velléitaire expectative, d'attente frustrée de quelque chose qui ne s'était jamais produit " (p. 1110). En tirant ce bilan négatif, il est moins question de vouloir amender un caractère inerte que de se préparer à accueillir la venue imminente d'une confirmation attendue, d'une chose trop bien entendue.

Dans la foulée survient l'idée que la chose (la cause) « était maintenant en train de se produire" (ibid.). Ce « quelque chose ", cette inconnue se résout par une double opération: la précipitation du moi et l'analyse du précipité qui contribue à en effectuer la dissolution (au fond, cette expérience produit des résultats analogues à la marche des trains qui rassemblent des troupes en désagrégeant le corps social). Sic transit gloria mundi. Dans le temps mort entre deux mouvements de locomotives, l'incertain répit "ménagé pour la première fois depuis le matin » (p. 1109) ne peut être que provisoire. Motivée par l'insignifiante inertie d'une cause résiduelle, la pensée comme les trains, ne cessera d'aller et venir dans le texte. 
Dans la camera obscura du wagon où se cristallise le personnage, le pronom de la non personne marque l'insignifiance du résidu. Comme dans un processus de développement photographique, les traces de différents déguisements, d'uniformes «successivement imposés ou essayés» (p. 1110) apparaissent en négatif et permettent de tirer le portrait du personnage, les postures d'un jeune dilettante: rigide uniforme de collégien, attirail d'apprenti peintre cubiste, nœud papillon, veston de tweed, chaussures en cuir de veau, vieux blouson de cuir pour aller à la pêche... À l'avenir, ces uniformes, rendus obsolètes par l'examen en cours, ne seront plus portés. Comme la syntaxe expulsée des manchettes, le sens esthétique, politique ou social des déguisements imposés ou essayés par le moi afin de lui permettre de s'afficher, les rôles qui l'ont affublé n'auront manifestement plus lieu d'être.

\section{PAPIER DE SOIE}

Dans les actualités désolantes d'une société en deuil, reste finalement le photogramme d'un geste délicat. L'empreinte d'une présence féminine pousse le personnage en retrait à s'avancer verbalement dans le récit en proférant son émotion par un juron:

et lui jurant de nouveau silencieusement, pensant: C'est ce papier; si au moins elle ne les avait pas emballés dans ce putain de papier de soie, ou de riz, ou je ne sais pas comment ça śappelle! [...] ce papier à demi transparent dans lequel la femme les avait enveloppés avec soin (p. 1113)

Avant de pouvoir l'ingérer, le voyageur mastique difficilement le viatique préparé et soigneusement emballé par sa compagne ${ }^{22}$. Cependant, l'en-cas ou plus exactement la demi-opacité de l'emballage transite mal. Tout en préservant l'autre moitié de cette provision, la parole bute sur un mot qui se trouve au bout de la langue; elle échoue à trouver le terme exact qui permettrait au personnage de saisir ce qui est en train de (lui) arriver. Un accroc dans le tissu appelle un art des coutures. Mais, en l'occurrence, cet art du faufilage, il (lui) reste encore à le trouver.

Avec ce trou de mémoire, c'est tout le tissu de la langue qui menace de s'effiler et le corps du voyageur se rétracte "Comme une huître sous le citron, pensa-t-il » (p. 1112). En relatant directement sous forme d'une excla-

22. De soie, de riz ou de journal, le papier emballe une nourriture substantielle: les gardes-mobiles, qui surgissent dans le compartiment comme enfantés de la nuit au cours du trajet mobilisateur, mastiquent tout à la fois machinalement et animalement leur casse-croûte enveloppé des pages aux gros titres noirs des journaux (p. 1131). 
mation silencieuse la réaction du sujet, le récit le mobilise à examiner la texture délicate d'une feuille de papier, en l'incitant à faire retour sur soi-même. Et plus exactement encore à revenir sur le sens du projet d'un apprenti en peinture et en littérature; un projet dans lequel une jeune femme prévenante tenait les rôles de modèle et de typographe. En l'absence du corps de celle " qui dormait à côté de lui » (p. 1113), entre veille et sommeil, le sujet examine sous un nouvel angle et de manière critique les efforts artistiques d'un étudiant en cubisme occupé à " étaler des couleurs sur ce qu'il appelait (ou essayait de se persuader qu'on pouvait appeler) des tableaux" (ibid.), à convertir (dans tous les sens du terme ${ }^{23}$ ) «sous formes d'ineptes rectangles, d'ineptes carrés ou d'ineptes pyramides (ou cônes, ou sphères, ou cylindres) les voiles, les barques et les rochers du port de pêche où ils passaient l'été (ou les seins, les cuisses, le ventre, la tendre chair respirante) " d'une jeune femme disposée à taper "les pages de ce qu'il se figurait que devait être un roman " (ibid.). À l'évidence, des accessoires comme machine à écrire, pinceaux, boite à couleurs n'ont plus qu'à être remisés dans ce compartiment, dans cette boite funéraire. Ces lignes de réflexions sans complaisance du sujet sont ici prises en charge et précisées par la forme du psycho-récit $\left(\mathrm{Cohn}^{24}\right)$ mais, dès l'étape lyonnaise, les trois modes de la "vie psychique " ne cesseront de dynamiser le récit, au moins jusqu’à la fin de la sixième partie.

Et cette dynamique reste indéfectiblement liée à la recherche d'un art que l'écriture ne cesse de problématiser. C'est non seulement l'écœurante et indigeste ineptie des efforts d'un apprenti qui se trouve mise en cause par un défaut de langue mais c'est, plus encore peut-être, la tenue (le principe directeur) d'une telle recherche artistique (son mur porteur) qui s'avère esthétiquement lézardée ${ }^{25}$.

Du papier de soie, qu'est-ce qu'elle s'imag..., la pâteuse et gluante boule de pain et de jambon mastiqués de la consistance maintenant d'une coulée de ciment refusant de descendre: Même si je me fourrais les doigts dans la gorge, pensa-t-il, jurant encore, comme si la colère (ou plutôt les simulacres de la colère et de la grossièreté) constituait à présent le seul recours (p. 1113-1114)

23. En particulier au sens bancaire de ce verbe, si l'on se souvient que c'est en signant des chèques que l'étudiant a pu voyager. Dans l'examen en cours, c'est la valeur de la signature apposée sur des œuvres autographiques, sur les titres de transport comme sur des factures qui peut être considérée comme un " faux en écriture " commis par un personnage trichant à moitié avec lui-même (p. 1128).

24. D. Cohn, op. cit., p. 37 sq.

25. Dans Soi-même comme un autre (Le Seuil, « Point Essais », 1990), Paul Ricœur pour sa part fait du caractère et de la parole tenue (d'une promesse, d'un engagement que l'on s'est fait) deux critères essentiels de l'identité personnelle. Cet engagement se trouve mis à mal par l'examen en cours dans L'Acacia. 
Le personnage pensif a beau mastiquer les morceaux, le mastic ne suffit plus à cimenter les fragments, comme si tout cela pouvait encore tenir ensemble. Au fond, c'est le papier de soi qui ne passe pas. Et il appartient au travail de la pensée d'en dissoudre les scories résiduelles, comme Pénélope défait la toile qu'elle vient tout juste de tisser.

Dans un essai sur l'identité personnelle, Charles Larmore amorce la question du moi en remettant sur le métier les notions un peu convenues d'authenticité et de sincérité ${ }^{26}$. À ce moment où nous devons également prendre en compte le simulacre d'émotion engagé d'un personnage trichant à moitié avec lui-même, que faire avec l'autre moitié qui continue d'avancer ${ }^{27}$ ? Prise dans le mouvement locomoteur, la pensée est contrainte de continuer (comme les personnages beckettiens) et de maintenir son allure contemplative en "pensant alors à penser d'urgence à autre chose ${ }^{28} . .$. " (p. 1114)?

Mais au fond, où pourrait-elle se diriger, cette pensée, lorsqu' elle veut penser à autre chose, alors que c'est la locomotive qui en règle le mouvement? Ce sont d'autres trains, d'autres déplacements aux confins de l'Europe (en plein casus belli) qui, par association, passeront par l'esprit du personnage: trains germaniques, polonais et soviétiques pris en 1937 par deux étudiants en cubisme, train espagnol en 1936 lors du passage de la frontière d'un jeune héritier (comme dirait Bourdieu) muni d'un "faux en écriture ». Ces déplacements babéliques méritent le détour d'une lecture minutieuse ${ }^{29}$. Le passage des frontières est l'un des thèmes insistants des déplacements analeptiques de ces deux jeunes apprentis «tout autour d'un continent trop vieux, malade, résonnant de bruits de bottes et de salves de pelotons » (p. 1110). Sans oublier celui de l'inspection d'une valise: un ballot qui semble diriger le voyageur plus qu'il ne la transporte ${ }^{30}$. Et, comme il se doit dans un tel emballage, on trouve en abondance des paperasses personnelles: chéquiers, photos d'identités réalisées par un photographe juif, papiers d'identité, invitations, carte timbrée du

26. Charles Larmore, Les Pratiques du moi, Le Seuil, 2004.

27. En particulier, avec l'autre moitié du viatique qui comme les chèques et la carte du parti (un faux en écriture) se trouve examinée comme titre de transport.

28. Un type de formule bien dans le style de l'oncle Charles qui, dans Histoire, aide le narrateur dans ses traductions latines: " pendant combien de temps as-tu fait semblant de faire semblant? (Hist., II, p. 173).

29. Jean-Yves Laurichesse l'effectue parfaitement dans "Tout autour d'un continent trop vieux: voyages en chemin de fer et nouveau roman d'apprentissage dans L'Acacia de Claude Simon ", dans Paul Carmignani (dir.), Bouleversants voyages: itinéraires et transformations, PUP, 2000, p. 377-395.

30. La valise aux étiquettes multicolores de l'étudiant (p. 1110); la valise de l'officier furibond (p. 1111); l'inspection des valises par les fonctionnaires soviétiques (p. 1115); l'examen du faux en écriture par les fonctionnaires espagnols (p. 1128); la valise des hommes qui attendent leur incorporation (p. 1146). 
parti, programmes, billets de banque... Sans oublier de vieux journaux avec lesquels se réactive l'énoncé prédictif inaugural légèrement modifié.

Et maintenant tout cela était loin, fini, et il allait mourir : à peine pourtant plus de deux ans depuis - deux ans à essayer de croire que ce qu'on pouvait lire à travers les articles des journaux n'arriverait pas, et sachant que cela ne pouvait pas ne pas arriver (p. 1127)

Dans ce contexte, la modification est notable. S'il s'agit d'indiquer l'imminence de la guerre (l'actualité d'une guerre annoncée depuis fort longtemps), quel sens donner à un adverbe et à un adjectif qui soulignent une distance, la rupture définitive entre deux époques: entre le présent (de la narration et de la fiction) et un passé définitivement révolu où cette nouvelle se trouvait alors déjà traitée et reçue de manière ambiguë, entre illusion et certitude? Dans l'univers simonien, nous le savons, la guerre est un événement immémorial, en permanence d'actualité. Le narrateur de La Corde raide n'en était-il d'ailleurs lui-même pas déjà informé? "Si on supprimait les trains, la guerre serait impossible. » Une hypothèse tout de suite corrigée: "Quelle bêtise! Comme si on ne se battait pas avant qu'il y eût des trains " ( $C R$, p. 146).

Au milieu du chemin de sa vie ("Nel mezzo del cammin di nostra vita" annonce Dante au seuil de son Enfer), en franchissant l'illusoire espace de vide tracé entre le quai et les wagons, le réserviste est entré dans un processus de transfiguration qui initie une autre temporalité que celle du temps commun des horloges. En témoigne cette exclamation silencieuse: "Mais que nous étions jeunes! Bon Dieu, que nous étions jeunes, que nous étions jeunes!...»(p. 1127 ; voir aussi p. 1117-1119), une manière de prendre acte de l'« irrémédiable coupure " (p. 1117) qui opère en permanence dans le texte. "Trois ans. Et maintenant sans doute avait-il tout de même fini par s'endormir " (p. 1130). En prenant le relais, le récit maintient la pensée en activité, en la tractant comme une locomotive vers un autre plan perceptuel (Deleuze). C'est du moins ce que suggère cette sorte de rêve éveillé où des gardes-mobiles fabriqués par la nuit traversent le compartiment. Comme si l'irruption d'une telle compagnie dans le texte venait matérialiser " en même temps que l'air frais du dehors une odeur de désastres " (ibid.), comme si ce mythique et fabuleux assemblage pouvait figurer le mouvement général de métamorphose (le devenir animal, végétal, minéral des hommes mobilisés) que le train de la mobilisation effectue très littéralement.

Revêtu du même blouson qu'autrefois, le personnage termine son voyage " étendu comme un cadavre, mais les yeux grands ouverts, glissant horizontalement dans la nuit» (p. 1128). Loin d'être un simple ornement stylistique, 
la comparaison demande à être prise au pied de la lettre: dépouillé de ses oripeaux de réserviste, le personnage aura fini par revêtir «la couleur des morts ", selon la formule de l'oracle de Delphes, donnée en réponse à la question taraudante ("Comment savoir? comment savoir?") de ceux qui comme Zénon de Kition semblent s'écarter de la voie (de la syntaxe) commune du langage ordinaire (des quotidiens ${ }^{31}$ ). À l'intérieur du dispositif spéculatif de l'écriture et jusqu'au bout du trajet, le moribond aura constamment pu être maintenu dans le texte dans une constante attitude contemplative. Son voyage nocturne dans la langue du récit inverserait l'épigraphe de Léonard de Vinci placée en tête de La Route des Flandres: "Je croyais apprendre à mourir, japprenais à vivre ${ }^{32}$. " Le contre-sens se prolongera jusqu'à la fin de la guerre.

\section{MOBILISATION GÉNÉRALE}

Au moment de disparaître du récit, le personnage demeuré seul (dans son rôle de réserviste) se réveille à nouveau avant de porter un autre masque, d'arborer un autre emploi sous l'uniforme d'un brigadier. Au demeurant, la singulière posture contemplative d'un sujet mobilisé - qui tour à tour veille et s'endort (le " tantôt je suis, tantôt je pense » de Valéry ${ }^{33}$ ), qui triche mais à moitié avec lui-même, qui est couché immobile dans un train en mouvement, - pourrait nous rappeler l'attitude paradoxale des cavaliers de la grande tradition lyrique, comme celle de Guillaume IX d'Aquitaine:

Je ne sais à quelle heure je suis né: je ne suis ni allègre ni irrité, je ne suis ni hors de moi ni à moi, et je n'en puis rien dire; car tel je suis frappé du destin [fadatz] la nuit sur une haute montagne. Je ne sais quand je me suis endormi, ni quand je m'éveille à moins qu'on ne me le dise ${ }^{34}$.

31. Selon Gilles Deleuze et Félix Guattari, les philosophes ne sont pas les seuls à revêtir cette couleur: "Le philosophe, le savant, l'artiste semblent revenir du pays des morts " (Qu'est-ce que la philosophie?, Minuit, 1991, p. 190). Quant à cette parole oraculaire, Hannah Arendt y fait allusion dans ses derniers travaux consacrés à la question de l'activité de la pensée (v. La Vie de l'esprit, trad. L. Lotringer, PUF, 1981 et Considérations morales, trad. M. Ducassou, Payot \& Rivages, coll. «Petite Bibliothèque ", 1996). Pour ce qui concerne la pensée romanesque à l'œuvre chez Claude Simon, voir les travaux de Philippe Sabot: Littérature et guerres, Sartre, Malraux, Simon, PUF, coll. "Lignes d'art ", 2010 et " Pratiques d'écriture et pratiques de pensée. Le cas de Claude Simon ", http://stl. recherche.univlille3.fr/seminaires/philosophie/macherey/Macherey20002001/Sabot.html.

32. «Je croyais apprendre à vivre, j’apprenais à mourir " ( $R F, \mathrm{I}, \mathrm{p} .195)$.

33. Paul Valéry, Euvres, Gallimard, «La Pléiade», 1957, t. I, p. 916.

34. Guillaume IX, «Fare un vers de dreyt nien » [Je ferai un vers sur pur néant], cité par Roger Dragonetti dans "La musique et les lettres". Etudes de littérature médiévale, Genève, Droz, 1986, p. 188 (trad. 
Dans L'Acacia, les manchettes des journaux remettent en œuvre cette $f a$ daise, en donnant à l'écriture l'occasion de déplacer une sottise singulière.

Dans le huitième chapitre, la mobilisation continue d'aller son train dans les transports en commun ainsi que dans la syntaxe des journaux. L'une comme l'autre uniformisent l'existence de chacun, reconfigurent « $[\mathrm{u}]$ ne petite foule » (p. 1146) impersonnelle en troupes revêtues d'un nouvel uniforme, transforment un réserviste en brigadier. Voici que ce dernier se trouve à nouveau « en train de coudre sur les manches de sa vareuse les deux chevrons de laine bleue qu'on lui avait attribués de force» (p. 1147) en compagnie de deux autres cavaliers. Le déguisement est neuf mais il reste parfaitement reconnaissable. Le costume demande encore à être étrenné par un nouveau personnage "aux prises non seulement avec son laborieux travail de couture" (p. 1149) mais une fois de plus avec la pensée de vingt-six années d'inexistence. Comme la démesure typographique, la cadence de la guerre se précipite. Au fil de mouvements ferroviaires proleptiques et analeptiques, les choses se dérèglent en même temps que l'écriture s'ingénie à reprendre et à monter soigneusement ce dérèglement syntaxique. Les moments contemplatifs, quant à eux, se feront de plus en plus rares.

Lors d'une nouvelle halte, le convoi militaire croise un express d'où descendent des civils, parmi lesquels une petite famille arborant des masques à gaz en bandoulière " comme ils auraient porté des épuisettes ou des cannes à pêche " (p. 1155). Ce croisement de deux catégories de train prend une tournure carnavalesque lorsque des hommes sur le pied de guerre se découvrent soudain débraillés dans le regard des civils qui les considèrent "avec une muette consternation" (ibid. ${ }^{35}$ ). L'un de ceux-ci exhibe "vers les braillards, un journal dont la manchette portait cette fois en lettres si énormes que chacun des deux mots emplissait toute la largeur de la page (et l'ensemble presque sa hauteur): MOBILISATION GÉNÉRALE »(ibid.). Désormais, l'événement (à la une des journaux du monde entier) peut être affiché en toutes lettres. Entre muette consternation et braillements militaires, c'est au récit qu'il revient de relater l'accomplissement de la mobilisation générale indiqué par le geste vindicatif et le regard mémorable d'un voyageur,

R. Dragonetti; le mot fadatz s'entend au sens du don des fées ou des Muses qui, comme expérience de l'altérité ne revient pas à la simple question convenue de l'inspiration).

35. Sur le plan initial d'une gare du Midi, au départ de la locomotive, un train des boulistes de retour d'un championnat vient se ranger à côté du train des réservistes « [c]omme par dérision [...] ou pour augmenter la confusion » (p. 1103). 
de même qu'ils avaient enregistré, vaguement ahuris mais sans véritable surprise, peutêtre même sans en comprendre tout à fait le sens, l'énorme manchette en lettres de deuil, comme un faire-part (celui de leur propre mort), qui s'étalait sur toute la longueur de la première page du journal déployé quelques heures plus tôt devant leurs yeux. (p. 1157)

De manière générale, la démesure d'un faire-part en lettres de deuil semble être reçue sans surprise; cette "sentence pour leur signifier leur bannissement» (p. $1163^{36}$ ) ne provoque qu'un vague ahurissement de la part des cavaliers. Rejetés de la communauté, sacrifiés, progressant mécaniquement sous la pluie, ceux-ci désormais sont en train de retourner à la boue originelle, comme le brigadier semble en avoir l'intuition (p. 1158). Mais sur un autre plan, le déploiement d'une manchette demeure longtemps parfaitement mémorable puisque

par la suite il (le brigadier) devait se rappeler cet homme debout, le journal déployé cachant son visage dont on ne voyait apparaitre au-dessus de la manchette que les deux yeux qui le fixaient avec une sorte de fureur, de reproche et de vindicative méchanceté. (p. 1155)

Comme le tohu-bohu des valises d'un officier furibond qui se répercutent sur les parois des wagons, le regard furieux de cet homme ${ }^{37}$ s'imprime dans la mémoire comme une citation (au sens tauromachique que lui conférait Barthes, comme le geste du torero incitant la bête à réagir ${ }^{38}$ ). En ce sens, elle implique les aiguilles, les poinçons, les stylets, les stylographes et tous les outils susceptibles de desceller la maçonnerie de l'« invisible muraille », propres à repriser «l'irrémédiable coupure » constamment présente dans un transport monumental et aérien. Sur la surface d'une « mince feuille de papier aux caractères monumentaux pour ainsi dire maçonnés» (p. 1163) se déploie dans l'écriture la puissance impersonnelle d'une singularité au plus haut point.

36. Un bannissement que commente Jean H. Duffy dans une perspective anthropologique, en y retrouvant une forme d'exclusion où le social se sépare rituellement d'une catégorie déterminée de ses membres: «Rite, passage, liminarité dans l'œuvre de Claude Simon ", Claude Simon, Allées et venues, éd. cit., p. 120 et sq. Dans Les Géorgiques ( $G$, p. 695 sq.), c'est dans la parenthèse de "l'incident du train des voyageurs " que s'effectue ce rituel.

37. Sans oublier cet autre geste d'un homme dans une cohorte de réfugiés chassés de chez eux " en même temps que les journaux imprimaient leurs énormes manchettes ", qui vitupère contre une colonne de cavaliers en mouvement "dans une langue, rauque, gutturale " des mots incompréhensibles, des mots "qui ressemblaient eux aussi à quelque chose de boueux, primitif, lourd, c'est-à-dire comme une ébauche de langue, un patois " (p. 1162).

38. Roland Barthes, S/Z, Le Seuil, coll. «Tel Quel», 1970, p. 29. 


\section{«...À L'INTENTION DE MYOPES OU D'IDIOTS »}

Le journal avec "l'ensemble de ses titres emphatiques " est un typique motif simonien. Voué à assurer "la flasque et plate oraison funèbre des évènements morts " ( $P$, I, p. 498), le journal s'avère être un média esthétiquement bien qualifié pour transmettre, dans toute sa banalité et dans toute sa trivialité, la violence permanente de l'existence, « les fragments de mots, d'images, arrachés à un monde violent, déclamatoire, et enfermés dans des rectangles bordés de noir, comme des faire-part de deuil " $(C C, \mathrm{II}$, p. 512). Le flux des faits divers qui font leur lit dans la presse quotidienne en abreuvant notre soif d'information, la syntaxe des nouvelles qui font les gros titres sont souvent associés à des séquences de restauration. Comme, par exemple, le journal du matin accompagnant les croissants du petit-déjeuner dans Le Vent ou La Route des Flandres, comme le journal appuyé sur une carafe sur la table d'un restaurant dans Histoire ou comme la page déchirée tenant lieu de nappe lors du repas de deux ouvriers dans Leçon de choses. Quant à la syntaxe des articles, le narrateur du Vent peut la décrire comme un ciment bouche-trou " apte à tous usages ", employé à " coller tant bien que mal ensemble " les morceaux de notre expérience par définition partielle, hétérogène et fragmentaire des choses; une sauce béchamel destinée à rendre comestible la brutale et indigeste matérialité d'un monde chaotique, " grâce à quoi (au grammairien, au rédacteur de service et à la philosophie rationaliste) chacun de nous peut avaler tous les matins, en même temps que les tartines de son petit déjeuner, sa lénifiante ration de meurtres, de violences et de folie» $(V, \mathrm{I}, \mathrm{p} .137)$.

Dans le monde simonien ${ }^{39}$, la « lecture du journal semble [...] se limiter à un coup d'œil rapidement jeté aux manchettes les plus voyantes. Encore cet acte minimum de lecture est-il rarement le résultat d'une volonté de lecture bien affirmée de prendre connaissance des nouvelles ", comme le remarque Brigitte Ferrato-Combe dans une étude très documentée de ce motif ${ }^{40}$. Si,

39. Voir l'analyse que Vincent Descombes fait de la lecture ordinaire des titres: "Comprendre qu'un fait est une nouvelle revient à comprendre ce que ce fait va changer dans notre monde " (op. cit., p. 162.). Sa conclusion montre bien que le lecteur simonien, lui, ne croit pas que quelque chose puisse fondamentalement changer dans le monde.

40. Brigitte Ferrato-Combe, "Fragments de journaux dans les romans de Claude Simon », dans F. Bassani, F. Chenet-Faugeras et D. Bougnoux (dir.), Journaux et journalistes, hommage à Jean Sgard, Recherches \& Travaux, n 48, Grenoble, université Stendhal-Grenoble 3, 1995, p. 156. Sur la question du journal et des journalistes, voir également Patrick Suter, " "Reportage" et "écriture" chez Claude Simon (Les Géorgiques et Le Jardin des Plantes) ", Études littéraires, vol. 40, 2009, p. 141-155. 
en effet, les titres sont généralement vite consommés et les pages des journaux aperçues furtivement, c'est que, dans l'économie du texte, l'usage des manchettes répond à des besoins élémentaires plus fondamentaux, à des fonctions vitales qui intéressent une esthétique de la digestion et du déchet. Il est en effet dans l'ordre des choses que les pages blanches des journaux et leur « terne, monotone et grisâtre alignement de menus caractères » où vient déchoir toute "l'agitation du monde", se périment rapidement. Mais ces déchets pourront encore servir à emballer des aliments, comme des bottes de poireaux, avant d'échouer froissés, "détrempés et à demi déchiquetés, sur l'eau sale des ports » (CC, II, p. 512). Peut-être même ces déchets trouveront-ils un nouvel emploi dans un art du recyclage, dans une esthétique des restes ${ }^{41}$, comme le suggère le narrateur photographe du Vent, "lorsque le regard tombe par hasard sur la feuille déchirée [...] et qu'alors, par la magie de quelques lignes tronquées, la vie reprend sa superbe et altière indépendance, redevient ce foisonnement désordonné, sans commencement ni fin, ni ordre " $(V, \mathrm{I}, \mathrm{p} .137)$. Ces lignes d'un programme poétique (régulièrement commentées), sont prises dans le récit d'un narrateur intrigué par le comportement surprenant de Montès, un personnage considéré socialement comme un idiot.

L'idiot est un autre motif simonien caractéristique. Comme le montre Aude Michard, cette œuvre traite les figures de l'idiot sous divers angles (sociologique, psychologique, thématique, dialogique, syntaxique ${ }^{42}$ ). Entre autres aspects, cette étude souligne judicieusement dans Le Vent la captieuse fascination du narrateur qui semble faire sa propre affaire de l'idiotie de son personnage. À ce cas intéressant, on ajoutera celui de $\mathrm{La}$ Corde raide où c'est déjà l'image d'un idiot que (sous la forme d'une comparaison) le miroir tend au narrateur pour lui indiquer l'altération de sa propre figure, "végétale comme un tronc d'arbre, un idiot, un train» (CR, p. 170). Dans L'Acacia, les transports en commun réarticulent et remobilisent de manière magistrale ces deux motifs, avec les titres médiatiques et l'idiotie de leurs destinataires.

Au terme de ce voyage, au cours duquel l'identité du personnage se sera progressivement (comme chimiquement) dissoute (dans une boue généralisée), il semble donc plutôt vain de se demander qui pourrait bien se trouver

41. L'écrivain, on le sait, se reconnait dans une esthétique du recyclage telle que l'illustrent des artistes comme Louise Nevelson, Rauschenberg ou Dubuffet.

42. Aude Michard documente ces figures dans son analyse du Vent tout en envisageant l'idiotie comme une "introduction à une théorie de l'écriture, à un art poétique " ("Visages de l'idiotie dans Le Vent de Claude Simon ", Cahiers Claude Simon, n 5, PUP, 2009, p. 28). 
sous le masque d'idiots auxquels sont destinées les manchettes. En s'adressant à tout le monde, elles ne s'adressent au fond à personne en particulier. En atteste l'anonymat de l'un des usagers de ces transports militaires. Comme le remarque Mireille Calle-Gruber, la figure paternelle tutélaire qui prend l'un des trains de la mobilisation reste sans nom propre (et sans tombe) et voue de la sorte (par provision) le sujet d'une quête biographique à prendre la forme de celle de personne ${ }^{43}$. Autrement dit, nous avons besoin d'une autre question catégorique pour caractériser la manière dont la démesure des lettres d'un titre s'imprime dans la mémoire, mobilise la pensée, le style d'un geste parfaitement idiot: une écriture.

\section{UNE CRÉATION CONTINUÉE}

Mais avant que la venue d'une "nuit tiède " de printemps puisse offrir une leçon de clarté, en attendant que les feuilles d'un acacia " comme animées soudain d'un mouvement propre, comme si l'arbre tout entier se réveillait, s'ébrouait, se secouait » (p. 1249) puissent redonner le goût de vivre et d'écrire, quelques étapes restent encore à parcourir à un corps provisoirement (dé)mobilisé « toujours étendu dans le noir comme les soirs précédents, sans autre désir lui semblait-il que celui que peut éprouver même pas un animal mais une mécanique, quelque chose comme une automobile après une longue course ou une locomotive ramenée au dépôt" (p. 1226). Telle une figure christique, c'est au "troisième jour seulement " que le sujet peut à nouveau être réveillé. Sur la voie de la geste testamentaire, ce réveil d'une locomotive demeure cependant parfaitement intriguant entre les lignes du roman d'apprentissage. Les pensées d'une mécanique refroidie sont aiguillées par le récit qui prend en charge le moment où, dans la grande maison familiale, le dépôt contemple les portraits, "les visages distants, bienséants, vaguement réprobateurs, de l'homme au fusil et de la femme démasquée, les deux lointains géniteurs qui deux cents ans auparavant s'étaient accouplés dans un froissement de linges troussés " (ibid.). Ce lointain couplage de deux portraits mobilisateurs semble maintenant hors de portée d'un simple

43. Autrement dit l'autobiographie de l'écriture. Mireille Calle-Gruber envisage même que "le geste de l'écriture peut se lire, en quelque sorte, geste de Dieu. Puisque ce n'est personne " (Le Grand Temps, Essai sur l'ouvre de Claude Simon, Lille, PU du Septentrion, 2004, p. 158). Dans une perspective critique, l'idiotie serait le symptôme de l'identité du "sujet moderne » comme "lieu constitutif de la réalité ", si l'on suit la lecture de Serge Margel dans "Beati pauperes spiritu, prolégomènes pour une topologie de l'unique (Matthieu, V, 3 et Jean, XIV, 3) ", dans Véronique Mauron et Claire de Ribaupierre (dir.), Les Figures de l'idiot, Rencontres du Fresnoy, Léo Scheer, 2004, p. 168 sq. 
télescope. En tout cas, la narration peut trouver bon de souligner qu’ « il lui fallut donc un moment pour comprendre ce qui ce soir-là le tenait éveillé dans le noir, empêchait la ténébreuse chape du sommeil de l'engloutir " (p. 1226), ou encore ce par quoi l'on entreprend de garder les yeux ouverts, en précisant que

ce qu'il éprouvait à présent, ce qui tenait ses yeux ouverts, ce n'était plus cette allégresse, ce vindicatif sentiment de triomphe et cette vindicative indignation à la pensée de ce qu'on lui avait fait ("La chèvre!", raconta-t-il plus tard (plus tard seulement: quand il fut à peu près redevenu un homme normal - c'est-à-dire un homme capable d'accorder (ou d'imaginer) quelque pouvoir à la parole, quelque intérêt pour les autres et lui-même à un récit (p. 1226-1227)

Dans ce processus se mélangent deux évènements distincts, deux temporalités fondamentalement différentes. La joie des premiers mots bredouillés et l'usage retrouvé des pouvoirs de la parole (dans les bras d'une "poule " aux cheveux rouges comme les yeux cerclés des mécaniciens de la locomotive) s'accomplissent avec l'idée d'une revanche. En retrait de toute parole possible, la puissance d'une pulsion précipite le dépôt (comme un chien) hors de la demeure familiale encombrée de légendes: " chèvre, loup et chasseur maintenant sortis de son esprit» (p. 1227) le projette hors des draps (de soie?) dont il est enveloppé. Le corps nu, intact, mais prisonnier du « même corps dans lequel il avait dormi depuis cinq mois, allongé chaque soir sur la mince paillasse d'une couchette » (p. 1224), se trouve maintenant moins ému que tiré, déporté par "quelque chose d'aussi furieux, d'aussi élémentaire et d'aussi impérieux que la faim ou la soif » (p. 1227). Avec le rejet de ces draps se nouent la fuite d'un prisonnier (un épisode antérieur diégétiquement) que l'écriture s'apprête à développer et la sortie de "l'esprit " (mais au juste lequel) d'une cohorte d'animaux fabuleux, au moment de filer furtivement vers un bordel de deuxième ou troisième zone. Qu'importe la cohérence illusoire d'épisodes séparés ${ }^{44}$. Ce geste est vital et convoquera un geste poétique de montage pour répondre de l'expérience et de l'évidence d'une radicale différence ontologique entre deux temporalités: le temps de disparaître dans un train (la disparition d'un personnage) et le temps de comprendre (com-prendre) en écrivant au présent l'événement disséminateur d'une mobilisation (historique et personnelle).

Entre un point de départ quelconque (mausolée, gare, centre mobilisateur...) et un point de retour, dans l'intervalle entre deux tronçons, dans

44. En tout cas pour un écrivain exploitant les tensions inhérentes à la différence absolue entre la discontinuité du monde perçu et la continuité de l'écriture ainsi qu'entre le temps de la langue (Tempus) et le temps extralinguistique (Zeit, pour parler dans la langue d'Harald Weinrich). 
l'espace illusoire de l'irrémédiable coupure entre deux wagons, peut se jouer la présence, le nunc stans d'une pensée, mobilisée par l'écriture à composer avec les bribes de mots décousus, de souvenirs, d'émotions, de sensations. Cette singulière forme de pensée se manifeste, par exemple, dans le monologue narrativisé où s'effectue une sorte "de fusion de deux en un ${ }^{45}$ ", où se croisent les fils du personnage et du récit. Dans le partage des voix se trouvent en permanence les marques discrètes d'une figure intermédiaire, la présence d'une "troisième voie ", d'un timbre ("mais comment dire? ", [p. 1227]), qui rappellent ce que Deleuze et Guattari pouvaient concevoir comme Personnage conceptuel. En art comme en philosophie, l'idiot (l'une des figures prises par les personnages des romans simoniens) fonctionne comme un embrayeur, un marqueur d'actes d'énonciation de la pensée ${ }^{46}$. Dans le train de l'écriture, ce personnage se retrouve convoqué dans le différentiel d'une pensée impersonnelle.

À plusieurs reprises dans des interviews, Claude Simon reviendra sur une conversation qu'il avait eue avec Merleau-Ponty, à l'issue du cours consacré par le philosophe à ses premières œuvres en 1961 au Collège de France, ainsi que sur son étonnement amusé de s'être découvert "rudement intelligent " (lui qui n'avait pas fait sa classe de philosophie) dans le commentaire du philosophe. S'il répète volontiers cette anecdote, c'est qu'il peut faire sienne la réponse de son lecteur. Le personnage que l'on suscite en travaillant n'est pas la personne de l'auteur. Indéterminable par principe (entre les pronoms de la personne et ceux de la non personne), l'intelligence du texte se déplace constamment, comme les trains, transportant la contemplation d'un voyageur insensible aux titres des manchettes, mais gardant les yeux ouverts sur leurs lettres; comme l'esprit d'un personnage, occupé à coudre des chevrons sur la manche d'un uniforme revêtant le vide de sa vie, est tiré par le fil de l'aiguille. C'est ainsi, dans L'Acacia, qu'en reprenant les lettres des titres pour les recomposer, la pensée tient le rôle de l'idiot et donne au passage un peu plus de forme au "ça pense" de La Corde raide (p. 170). À sa table d'écrivain, en avançant à tâtons dans la forêt des signes, Claude

45. Voir D. Cohn, op. cit., p. 136. Ce «deux-en-un» est une formule qui s'impose dans la conception arendtienne de la pensée. La narratologie contribue à éclairer, à " matérialiser ", dans une perspective linguistique, l'idée de la pensée (v. H. Arendt, La Vie de l'esprit, éd. cit., p. 235 sq.). De son côté, Bernard Andrès peut caractériser la recherche poétique simonienne comme "alterbiographie d'un ego recouvré ": on relira, en l'occurrence, avec profit son analyse du montage (au sens cinématographique) de la onzième partie de L'Acacia où il envisage le personnage comme dispositif (Profils du personnage chez Claude Simon, Minuit, 1992, p. 215).

46. G. Deleuze et F. Guattari, op. cit., p. 62. 
Simon aura réussi à installer les formes d'une méditation dans l'avancement de son œuvre, en laissant le rythme d'une pensée scander son écriture, à préciser esthétiquement les formes d'une méditation.

Dans le travail de l'écriture, cette méditation esthétique intervient donc comme force de dissolution mais aussi bien comme force de composition. L'une et l'autre contribuent à effectuer l'événement de l'œuvre, engagent une recherche poétique pour répondre pratiquement de la question de l'art, au sens fort. Lécrivain ne trouve-il d'ailleurs pas opportun de rappeler: "Comme la science, l'art remplace l'idée absente de Dieu (ou du moins son silence...) il est pour l'homme, au milieu de l'écoulement universel des apparences, le seul absolu auquel l'individu puisse aspirer ${ }^{47}$. " En envisageant l'éventualité de la déficience d'un principe fondateur, d'une cause première, une pensée méditative (comme celle de Descartes, par exemple) se trouve convoquée à en tirer les conséquences. Ne serait-ce que pour s'essayer à procurer au sujet une idée (une image) ferme de sa durée, une durée menacée par une temporalité infiniment divisible où chaque moment présent " ne dépend en aucune façon des autres ${ }^{48}$ ». Le silence de Dieu n'est-il pas flagrant dans la paisible splendeur des paysages printaniers de L'Acacia? À l'issue d'une débâcle de sept jours, la mutation de candides et dociles enfants de troupes en " choses inertes " ne pourrait-elle être l'œuvre d'un Créateur qui aurait " employé ce temps à parfaire son œuvre, puis, facétieusement, à la détruire " (p. 1032) ? Sur le plan de cette cosmogénèse inversée, sont inscrites les traces d'une sacrée affaire (qui aura, entre autres, mobilisé ce " grand capitaine de l'esprit ", selon le qualificatif de Valéry $\left.{ }^{49}\right)$ : celle d'une création continuée ${ }^{50}$. Cette affaire est celle de la question du temps; et de temps, il en est précisément question dans la maintenance de la mobilisation. Mais Claude Simon ne prenait-il pas le plus grand soin à éviter le terme de création (et de ses implications onto-théologiques) en lui substituant celui de production $^{51}$ ?

47. " Écrire ", Quatre conférences, Minuit, 2012, p. 97.

48. Descartes, Méditations métaphysiques, Gallimard, "La Pléiade », 1937, p. 297.

49. Paul Valéry, Une vue de Descartes, Gallimard, "La Pléiade », t. I, 1957, p. 835.

50. Selon Fabien Revol, cette notion théologique prendrait ses racines dans la conception augustinienne du temps (v. "La création continuée en question ", dans F. Mies (dir.), Que soit! Lidée de création comme don à la pensée, coll. " Donner raison ", n 41, Bruxelles, Lessius, 2013, p. 293-303).

51. "J'aurais préféré dire production que création qui postule un processus ex nibilo, alors que l'écrivain travaille un matériau - le langage - chargé d'histoire... " (La fiction mot à mot, I, p. 1185, c'est Simon qui souligne). 
Comme l'on comprend la méfiance de l'écrivain pour les spéculations philosophiques! Toutefois, lorsqu'il parle du travail des peintres, n'est-ce pas justement ce terme de méditation qu'il emploie pour caractériser le travail des peintres (tel Cézanne)? "Toute production d'image s'élabore dans une durée, et précise-t-il, est le résultat d'une méditation, d'une addition et d'une combinaison de présents accumulés, même s'il s'agit d'un peintre non figuratif ${ }^{52}$. " Il faut ajouter que cette question de temporalité intervient également dans la réception de l'image, comme on le voit bien, par exemple dans Histoire avec la fameuse photographie de l'atelier du Hollandais, lorsqu'elle anime l'esprit du narrateur: «l'esprit (ou plutôt: encore l'œil, mais plus seulement l'œil, et pas encore l'esprit: cette partie de notre cerveau où passe l'espèce de couture, le hâtif et grossier faufilage qui relie l'innommable au nommé)»(Hist., II, p. 328). Cette couture esthétique est bien l'art d'une écriture du sensible. Dans la vision cinématographique ${ }^{33}$ de l'écrivain, la succession d' "images fixes " se produit dans la durée mais cette production ne peut s'accomplir sans trouver l'idée propre à combiner les présents qui differrent.

"Pourquoi écrivez-vous? " En réponse à cette question (plutôt) convenue, Simon répondait qu'il en allait sans doute d'un désir de reconnaissance sociale, mais peut-être plus essentiellement encore d'un besoin de « justifier sa propre existence devant soi-même, par un "faire” ". Tout en précisant:

ce n'est pas le " Cogito ergo sum » de Descartes, mais bien plutôt un: « je fais (je produis) donc je suis ", besoin, me semble-t-il, élémentaire et que ressent tout homme normal en y répondant sous une forme ou sous une autre, que ce soit en faisant venir une récolte, en faisant des affaires, en faisant ou construisant un pont, des machines, en faisant de la recherche, etc. ${ }^{54}$

Cette conception simonienne plutôt pragmatique d'une pensée incarnée procure au lecteur une expérience esthétique, au sens où un John Dewey par exemple pouvait concevoir l'art ${ }^{55}$. D'une manière ou d'une autre, la " pensée du roman " dans le texte simonien entraîne l'intelligence de la lecture, la mobilise à entreprendre en permanence une réforme de l'entendement ${ }^{56}$. Et, en effet, avec ponctualité, la locomotive de L'Acacia ne cesse de revenir mobiliser notre méditation.

52. Claude Simon, Photographies, Maeght, 1992, p. 17.

53. Une photographie (réelle ou fictive) décrite comme celle d'Histoire est un photogramme.

54. Claude Simon « Ecrire», op. cit., p. 76 (c'est Simon qui souligne).

55. John Dewey, L'Art comme expérience, trad. coord. par J.-P. Cometti, Gallimard, « Folio Essais », 2005.

56. V. Descombes, op. cit., p. 46. 